\title{
Upward and Downward Gaze Palsy, Convergence Palsy, Concomitant Skew Deviation and Bilateral Light-Near Dissociation Due to a Unilateral Rostral and Dorsal Midbrain Infarction
}

\author{
Hiromasa Tsuda $^{\text {a, }}$, Aya Maekawa ${ }^{\mathrm{a}}$, Masaki Ishihara ${ }^{\mathrm{a}}$
}

\begin{abstract}
A 48-year-old man with atrial septal defect abruptly developed upward and downward gaze palsy with a loss of vertical oculocephalic reflex and smooth pursuit, convergence palsy, concomitant skew deviation with left-sided hypotropia, and bilateral light-near dissociation. Cranial magnetic resonance imaging demonstrated a localized infarction in the left-sided rostral and dorsal midbrain. Neuro-ophthalmologic findings suggested that the left-sided rostral interstitial nucleus of the medial longitudinal fasciculus, left-sided interstitial nucleus of Cajal, and posterior commissure might be involved. There were not convergence retraction nystagmus, and eyelids retraction and lag, probably because the nucleus of the posterior commissure might be spared.
\end{abstract}

Keywords: Interstitial nucleus of Cajal; Parinaud's syndrome; Posterior commissure; Pretectal syndrome; riMLF

\section{Introduction}

There were not many reported cases of upward and downward gaze palsy due to a unilateral rostral and dorsal midbrain lesion [1-10]. Therefore, the neural structures and pathways underlying vertical gaze control are yet not fully understood. We herein describe a rare case of upward and downward gaze palsy with a loss of vertical smooth pursuit and oculocephalic reflex, convergence palsy, concomitant skew deviation, and bilateral light-near dissociation due to a unilateral rostral and dorsal midbrain infarction. However, ocular tilt reaction, convergence retraction nystagmus, and eyelids abnormalities were not observed. The etiology of these neuro-ophthalmologic symptoms was discussed in detail.

Manuscript accepted for publication October 22, 2015

aDepartment of Neurology, Tokyo Metropolitan Health and Medical Corporation Toshima Hospital, Japan

${ }^{b}$ Corresponding Author: Hiromasa Tsuda, Department of Neurology, Tokyo Metropolitan Health and Medical Corporation Toshima Hospital, 33-1, Sakaecho, Itabashi-ku, 173-0015 Tokyo, Japan.

Email: hiromasa_tsuda@tokyo-hmt.jp

doi: http://dx.doi.org/10.14740/jmc2349w

\section{Case Report}

A 48-year-old Japanese man with atrial septal defect abruptly complained of nausea, dizziness, and binocular diplopia in January 2015. Two days later, the patient came to our neuroophthalmology service and was admitted. No treatment has been performed for atrial septal defect. There was not any other contributory medical history. Consciousness was alert. The patient was found to have a blood pressure of $144 / 86 \mathrm{mmHg}$ and heart rate of $62 / \mathrm{min}$. General examination demonstrated no abnormalities. The diameter of the pupil was $5 \mathrm{~mm}$ bilaterally in the illuminated room. In both eyes, though pupil responses to direct and indirect light responses were sluggish, near response of pupil was prompt. Consequently, bilateral light-near dissociation was confirmed. Palpebral aperture measured 8.5 $\mathrm{mm}$ in both eyes. Eyelids retraction and lag was not observed. There was upward and downward gaze palsy. Upward gaze palsy was greater than downward (Fig. 1). Vertical oculocephalic reflex as well as smooth pursuit was not observed. In addition, convergence palsy and concomitant skew deviation with left-sided hypotropia were demonstrated (Fig. 1). Nystagmus was not observed. Bell's phenomenon was not detected in both eyes. Maddox rod test demonstrated normal torsion in both eyes. Abnormal head tilt was not observed. There were no other neurological abnormalities. Complete blood cell count and laboratory examination were within normal ranges. Chest roentgenogram, electrocardiography and carotid ultrasonography demonstrated no abnormalities. Transthoracic echocardiography demonstrated atrial septal defect and no thrombosis in the left atrium. Transesophageal echocardiography could not be performed, because of the patient's refusal. Computed tomography venography demonstrated no abnormalities in the lower extremities and lung. Cranial magnetic resonance imaging demonstrated a localized infarct lesion in the rostral and dorsal midbrain on the left side (Fig. 2). Cranial magnetic resonance angiography demonstrated no abnormalities. Regarding the etiology of left-sided midbrain infarction, paradoxical embolism secondary to atrial septal defect was suspected. Using 7 days of sodium ozagrel at $160 \mathrm{mg} /$ day, the patient did not complain of nausea and dizziness, and pupil function, downward gaze, vertical oculocephalic reflex, and vertical smooth pursuit were normalized. Consequently, the patient was discharged. Thereafter, under oral clopidogrel at $75 \mathrm{mg} /$ day, the patient became asymptomatic within a month. However, bilateral Bell's 


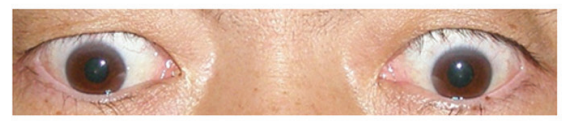

primary position in the illuminated room

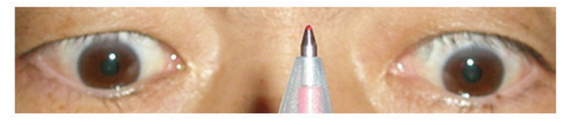

convergence

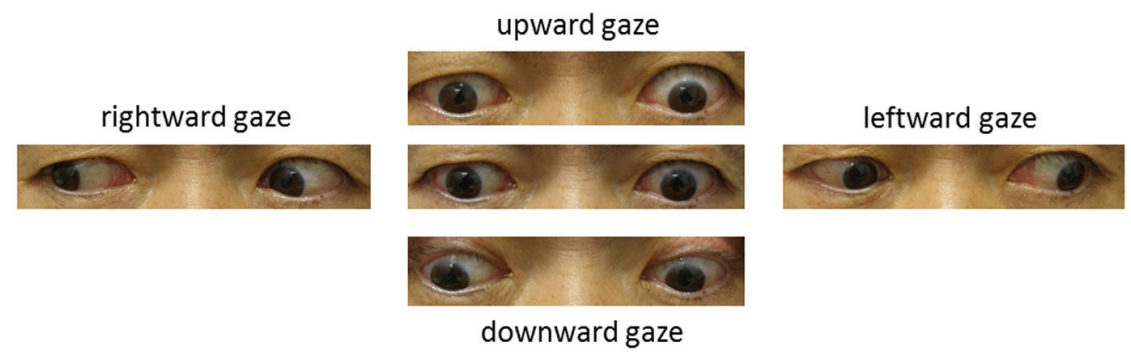

Figure 1. Pupil diameter was $5 \mathrm{~mm}$ in both eyes in the illuminated room. Palpebral aperture measured $8.5 \mathrm{~mm}$ in the both eyes (upper and left panel). Despite convergence palsy, near response of pupil was prompt in both eyes (upper and right panel). Upward and downward gaze palsy and concomitant skew deviation with left-sided hypotropia were observed. Upward gaze palsy was greater than downward (lower panels).

phenomenon was not observed.

\section{Discussion}

The rostral interstitial nucleus of the medial longitudinal fasciculus (riMLF) is a wing-shape nucleus, lying dorsomedial to the red nucleus and rostral to the oculomotor nuclei. It contains the neural generators for bilateral vertical saccades. Excitatory burst neurons within this nucleus send collaterals to motoneurons supplying yoked muscle pairs of the two eyes.
Axon collaterals responsible for upward saccades reach bilaterally the superior rectus muscle and inferior oblique muscle crossing within the oculomotor nucleus, whereas collaterals for downward saccades project only to the ipsilateral inferior rectus and superior oblique which act as depressor muscles. The interstitial nucleus of Cajal (INC) lies adjacent and caudal to the riMLF, and is the neural integrator for vertical and torsional gaze. It is responsible for the vertical smooth pursuit and vertical vestibular ocular reflexes. Neurons contained in this nucleus contribute to hold the eyes in eccentric gaze after a vertical saccade and in the eye-head coordination in the roll

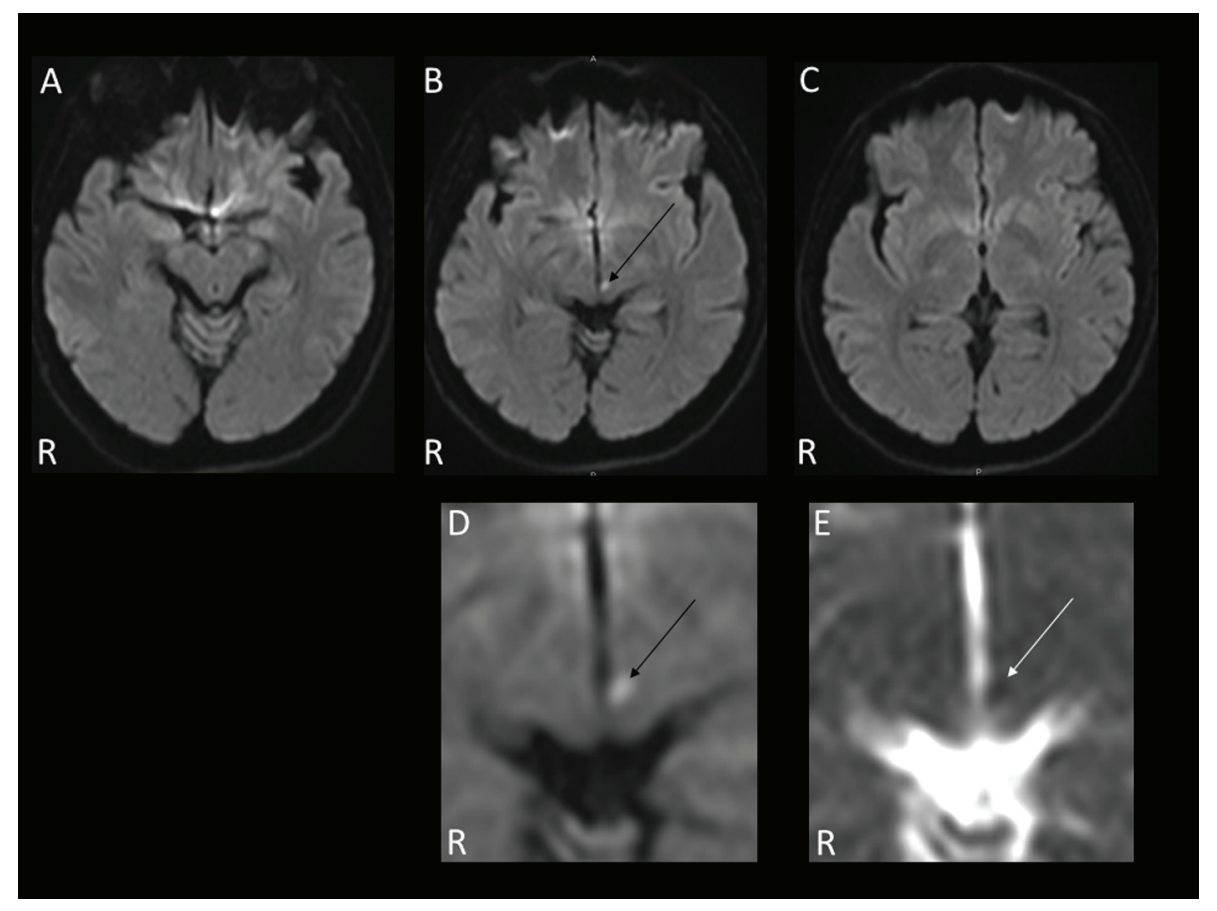

Figure 2. Cranial magnetic resonance imaging on axial image (A-D: diffusion-weighted, D: magnification of $B$, E: apparent diffusion coefficient) demonstrated a localized infarct lesion in the rostral and dorsal midbrain on the left side (arrows). 
plane [11]. Moreover, INC receives neuron from the vestibular nucleus. Therefore, INC involvement can cause ocular tilt reaction, which consists of skew deviation, conjugate ocular torsion and head tilt $[10,12]$. The posterior commissure contains the projections from INC to the contralateral oculomotor nuclei and the opposite INC. Furthermore, it contains axons responsible for upward gaze originating from the nucleus of the posterior commissure (NPC) and projecting to the riMLF, and to the INC. In addition, the posterior commissure contains the M-group, which is important for coordinating eyelid movements with vertical gaze. The NPC is lying rostral to the superior colliculi at the junction of the aqueduct and third ventricle [11]. However, in vertical gaze and eyelid movement, the role of the NPC remains unclear. Based on two cases of eyelid retraction and eyelid lag with minimal vertical gaze impairment, Galetta et al [13] noted that a premotor eyelid control might locate in the region of the NPC.

The reason why unilateral rostral midbrain infarctions can induce conjugate upward and downward gaze palsy remains uncertain. Bogousslavsky et al [1] reported an autopsy case of conjugate upward and downward gaze palsy due to a unilateral infarct destroying the riMLF, but sparing the posterior commissure and INC. The authors stated that a unilateral riMLF lesion may have disrupted bilateral upgaze excitatory and inhibitory inputs and unilateral downgaze excitatory inputs [1]. Ranalli et al [2] noted a case of paralysis of upward saccades, decreased amplitude and velocity of downward saccades and vertical smooth pursuit, as well as decreased gain and amplitude of the vertical ocular reflex. The neurophysiological examination demonstrated involvement of the right riMLF, a part of the right INC, and the NPC, but the posterior commissure tract was preserved. The authors hypothesized that downward gaze fibers might partly decussate in the ventral posterior commissure and transverse the region of the opposite riMLF [2].

The mechanism of vertical oculocephalic reflex and smooth pursuit is still controversial. Ohashi et al [10] described a case of ocular tilt reaction, and upward and downward gaze palsy with a loss of vertical smooth pursuit and oculocephalic reflex secondary to a unilateral midbrain hemorrhage. The authors noted that unilateral riMLF and INC might be involved, and damage to the unilateral INC might cause ocular tilt reaction. However, the relationship between involvement of INC and a loss of vertical oculocephalic reflex and smooth pursuit was not mentioned [10]. Thereafter, there were two reported cases of upward and downward gaze palsy with a loss of vertical oculocephalic reflex and smooth pursuit due to a unilateral midbrain infarction $[3,4]$. In both cases, convergence of eyes as well as Bell's phenomenon was preserved, and ocular tilt reaction was not detected. Although unilateral riMLF and INC were involved, the posterior commissure was spared. The authors speculated that unilateral INC involvement might cause a loss of vertical oculocephalic reflex and smooth pursuit $[3,4]$. The main projections from the INC to ocular motoneurons and the contralateral INC are through the posterior commissure. Consequently, a lesion of unilateral INC may be, in effect, a bilateral lesion, and so affects inputs to ocular motoneurons on both sides [11].

In human, the mechanism of convergence is still uncertain. Nashold et al $[14,15]$ reported that, in all of 16 patients, after the stereotactic mesencephalotomy for pain control, uni- lateral destroyed lesion which located below the superior follicular region in the vicinity of the posterior commissure caused convergence palsy.

The precise etiology of light-near dissociation remains uncertain. Based on the neuroimaging analysis, Shams et al [16] stated that pretectal lesion affecting the posterior commissure might cause bilateral light-near dissociation. Midline lesion of the posterior commissure interrupts most projections from both pretectal olivary nucleus, and may cause bilateral symmetric loss of the pupillary light reflex with preservation of the near response. The authors speculated that the near-reflex fibers approached the Edinger-Westphal nucleus through the ventral region, unlike the afferent retinal light-near reflex fibers that follow a dorsal route [16].

Convergence retraction nystagmus is characterized by fast convergence and retraction of the eyes with a slow divergence and forward movement of the eyes, and may develop in dorsal midbrain lesion. However, its pathological etiology remains unclear. Riodan-Eva et al [17] reported a case of convergence retraction nystagmus secondary to involvement of unilateral riMLF, rostral portion of the INC and NPC. Based on the electrophysiological analysis in a case of unilateral riMLF and NPC impairment, Rambold et al $[18,19]$ stated that convergence retraction nystagmus might be a disorder of vergence rather than of opposing adducting saccades.

In our patient, upward and downward gaze palsy might be caused by damage to the left-sided riMLF. In addition, loss of vertical oculocephalic reflex and vertical smooth pursuit might be induced by impairment of the left-sided INC. However, there was not ocular tilt reaction, despite involvement of unilateral INC. Therefore, we considered that, in our patient, the leftsided INC was partially damaged. Concomitant skew deviation is defined as vertical misalignment of the eyes, and a poorly localizing sign of posterior fossa dysfunction [20]. Convergence palsy might result from impairment of the region which located at the posterior commissure and lateral to the Sylvian aqueduct. Bilateral light-near dissociation might be induced by involvement of the posterior commissure. There were not convergence retraction nystagmus, and eyelids retraction and lag, probably because the NPC might be spared. We believed that our patient had no Bell's phenomenon in both eyes by nature. In our patient, vertical gaze palsy continued longer than the other neuroophthalmologic symptoms. Consequently, the left-sided riMLF might be damaged intensely, whereas the left-sided INC and posterior commissure might be partially involved. In conclusion, we emphasize that, in cases of unilateral rostral and dorsal midbrain infarction, detailed neuro-ophthalmologic examination is very useful for precise recognition of its extent.

\section{Conflict of Interest}

The authors state that they have no conflict of interest.

\section{Grant Support}

None. 


\section{References}

1. Bogousslavsky J, Miklossy J, Regli F, Janzer R. Vertical gaze palsy and selective unilateral infarction of the rostral interstitial nucleus of the medial longitudinal fasciculus (riMLF). J Neurol Neurosurg Psychiatry. 1990;53(1):6771.

2. Ranalli PJ, Sharpe JA, Fletcher WA. Palsy of upward and downward saccadic, pursuit, and vestibular movements with a unilateral midbrain lesion: pathophysiologic correlations. Neurology. 1988;38(1):114-122.

3. Pothalil D, Gille M. Conjugate downward and upward vertical gaze palsy due to unilateral rostral midbrain infarction. J Neurol. 2012;259(4):779-782.

4. Alemdar M, Kamaci S, Budak F. Unilateral midbrain infarction causing upward and downward gaze palsy. J Neuroophthalmol. 2006;26(3):173-176.

5. Rabadi MH. Neurological picture. Unilateral midbrain infarct presenting as dorsal midbrain syndrome. J Neurol Neurosurg Psychiatry. 2013;84(9):973-975.

6. Brigo F, Lochner P, Tomelleri G, Moretto G, Nardone R, Gursahani R. Bilateral vertical gaze palsy in unilateral mesodiencephalic junction lesion: a case series. Neurol India. 2013;61(2):170-173.

7. Beversdorf DQ, Jenkyn LR, Petrowski JT, 3rd, Cromwell LD, Nordgren RE. Vertical gaze paralysis and intermittent unresponsiveness in a patient with a thalamomesencephalic stroke. J Neuroophthalmol. 1995;15(4):230-235.

8. Seifert T, Enzinger C, Ropele S, Storch MK, Fazekas F. Midbrain ischemia presenting as vertical gaze palsy: value of diffusion-weighted magnetic resonance imaging. Cerebrovasc Dis. 2004;18(1):3-7.

9. Pierrot-Deseilligny CH, Chain F, Gray F, Serdaru M, Escourolle R, Lhermitte F. Parinaud's syndrome: electro-oculographic and anatomical analyses of six vascular cases with deductions about vertical gaze organization in the premotor structures. Brain. 1982;105 (Pt 4):667-696.

10. Ohashi T, Fukushima K, Chin S, Harada T, Yoshida K, Akino M, Matsuda H. Ocular tilt reaction with vertical eye movement palsy caused by localized unilateral midbrain lesion. J Neuroophthalmol. 1998;18(1):40-42.

11. Bhidayasiri R, Plant GT, Leigh RJ. A hypothetical scheme for the brainstem control of vertical gaze. Neurology. 2000;54(10):1985-1993.

12. Halmagyi GM, Brandt T, Dieterich M, Curthoys IS, Stark RJ, Hoyt WF. Tonic contraversive ocular tilt reaction due to unilateral meso-diencephalic lesion. Neurology. 1990;40(10):1503-1509.

13. Galetta SL, Gray LG, Raps EC, Schatz NJ. Pretectal eyelid retraction and lag. Ann Neurol. 1993;33(5):554-557.

14. Nashold BS, Jr., Seaber JH. Defects of ocular motility after stereotactic midbrain lesions in man. Arch Ophthalmol. 1972;88(3):245-248.

15. Seaber JH, Nashold BS. Comparison of ocular motor effects of unilateral stereotactic midbrain lesions in man. Neuroophthalmology. 1980;1(2):95-99.

16. Shams PN, Bremner FD, Smith SE, Plant GT, Matthews TD. Unilateral light-near dissociation in lesions of the rostral midbrain. Arch Ophthalmol. 2010;128(11):14861489.

17. Riordan-Eva P, Faldon M, Buttner-Ennever JA, Gass A, Bronstein AM, Gresty MA. Abnormalities of torsional fast phase eye movements in unilateral rostral midbrain disease. Neurology. 1996;47(1):201-207.

18. Rambold H, Kompf D, Helmchen C. Main sequence of convergence retraction nystagmus indicates a disorder of vergence. Ann N Y Acad Sci. 2002;956:592-594.

19. Rambold H, Kompf D, Helmchen C. Convergence retraction nystagmus: a disorder of vergence? Ann Neurol. 2001;50(5):677-681.

20. Keane JR. Ocular skew deviation. Analysis of 100 cases. Arch Neurol. 1975;32(3):185-190. 